\title{
Chain Length Dependencies of the Bending Modulus of Surfactant Monolayers
}

\author{
Live Rekvig, ${ }^{1, *}$ Bjørn Hafskjold, ${ }^{1}$ and Berend Smit $^{2}$ \\ ${ }^{1}$ Department of Chemistry, Norwegian University of Science and Technology, N-7491 Trondheim, Norway \\ ${ }^{2}$ Department of Chemical Engineering, University of Amsterdam, Nieuwe Achtergracht 166, 1018 WV Amsterdam, The Netherlands
}

(Received 22 October 2003; published 15 March 2004)

\begin{abstract}
The effect of the surfactant chain length $n$ on the bending modulus $\kappa$ of surfactant monolayers is simulated with a mesoscopic oil-water-surfactant model. We confirm a power law, $\kappa \propto n^{p}$, as predicted by mean-field theory and found experimentally, and find $p \approx 1.5$ at a constant surface density and $p \approx 1.0$ at a constant interfacial tension. This agrees quite well with both mean-field theory ( $p=2-3$, assuming constant surface density) and experiments (at constant surface tension). Our results suggest that the previously reported agreement between theory and experiment may be fortuitous and caused by the difference in surfactant types.
\end{abstract}

DOI: 10.1103/PhysRevLett.92.116101

PACS numbers: 61.20.Ja, 68.05.Gh, 82.70.-y

Sufficiently far from a critical point, the properties of an oil-water interface are governed by the interfacial tension. To minimize the surface free energy, the system adopts a configuration that minimizes the interfacial area. Adding surfactant can dramatically change the properties of the interface. Because of their amphiphilic character, surfactant molecules adsorb at the oil-water interface and reduce the interfacial tension. Depending on the surfactant structure, the interfacial tension can become so low that the free energy associated with changes of the curvature of the interface has to be taken into account to understand the properties of the interface [1]. For example, the formation of microemulsion and other phases in surfactant-oil-water systems are explained in terms of the bending modulus which characterizes the free energies of the interface related to changes in the curvature.

Whereas we do have a good understanding of how changes in the surfactant structure influence the interfacial tension, relatively little is known of how these changes affect the bending modulus of the interface. Only recently, the relation between the bending modulus and surfactant chain length has been addressed both experimentally, using high-resolution scattering techniques [2], and theoretically, using mean-field theories $[3,4]$. On the basis of these theories, the experimental data are interpreted [5-7] with a power-law dependence of the bending modulus $\kappa$ as a function of the chain length $n$ :

$$
\kappa \propto n^{p}
$$

Mean-field theories predict $p=2-3[1,8]$, but a recent study shows a strong density dependence [4]. The continuum elasticity theory gives $p=3$ [1], and experiments indicate $p \sim 3[5,6]$. However, most experiments measure only the combination $\kappa+\bar{\kappa} / 2$ where $\bar{\kappa}$ is the saddle splay modulus $[5,6,9]$.

The mean-field predictions of $p$ are based on comparison of surfactant monolayers of various chain lengths that have the same surface density $\left(N_{\text {surf }} / A\right)$. Experimentally, it is very difficult to measure or to control the area per surfactant. The experiments have been performed at similar (very low) interfacial tension. This implies that the surface density of surfactant varies with $n$ and therefore these experiments may not be compared directly with the theoretical predictions. This issue motivated us to study the bending modulus of surfactant monolayers using molecular simulations. In this Letter, we introduce a mesoscopic oil-water-surfactant model and use dissipative particle dynamics to compute the effect of changes in the surfactant chain length on the bending modulus both at a given interfacial tension and at a given surface density.

Computing the bending modulus in a molecular simulation is very CPU intensive since one has to analyze the fluctuations of the interface. This requires long simulations on a relatively large system [10]. The height fluctuations in the monolayer can be written in terms of wavelength dependent undulation modes via a Fourier transform. By assuming equipartition, we can relate these undulation modes for small values of the wave vector $q$ to the interfacial tension $\gamma$ and the bending modulus $[1,10]$,

$$
\left\langle|\tilde{h}(q)|^{2}\right\rangle=\frac{k_{B} T}{A} \frac{1}{\gamma q^{2}+\kappa q^{4}},
$$

where $k_{B} T$ is Boltzmann's constant times the temperature and $A$ is the projected area of the interface onto a plane parallel with the interface. This method has been used to compute the bending modulus of a biological membrane $[10,11]$ and a surfactant monolayer [12]. These simulations are important since they demonstrate the feasibility of the method. However, the data are focused on only a few configurations and therefore do not give us sufficient insight into the surfactant chain length dependence to test the theoretical predictions.

In our work, we use a mesoscopic oil-water-surfactant model $[13,14]$ in which oil and water are represented by spherical particles denoted $o$ and $w$, respectively. A surfactant molecule is constructed by connecting 
hydrophilic head particles $h$ and hydrophobic tail particles $t$ with harmonic springs. By changing the number of tail particles, we can study the effect of chain length on the properties of the system. We use dissipative particle dynamics (DPD) to simulate our system. In DPD, we distinguish three types of forces; random forces, dissipative forces, and conservative forces. The random and dissipative forces are chosen such that a canonical ensemble is sampled. We use the conventional forms which have been described extensively in the literature [15]. The conservative forces define the mesoscopic model. We use the commonly used soft-repulsive interaction model [16] to describe the forces between the $o, w, h$, and $t$ particles:

$$
\mathbf{f}\left(r_{i j}\right)=a_{i j} \hat{\mathbf{r}}_{i j}\left\{\begin{array}{ll}
1-\frac{r_{i j}}{r_{c}} & \text { if } r_{i j}<r_{c} \\
0 & \text { if } r_{i j} \geq r_{c}
\end{array},\right.
$$

where $r_{c}$ is the cutoff radius of the potential, $r_{i j}$ is the distance between particles $i$ and $j$, and $a_{i j}$ is the repulsion parameter that defines the model. These parameters are chosen such that oil and water particles do not mix and the head and tail particles are hydrophilic and hydrophobic, respectively $\left(a_{w w}=a_{o o}=25, a_{o w}=a_{o h}=80\right.$, $a_{w h}=15$, and $a_{h h}=35$, with tail particles identical to oil particles). The spring constant and equilibrium length of the harmonic spring connecting the surfactant segments are $k_{s}=100$ and $r_{0}=0.7$, respectively. These parameters are based on the work of Groot [16] and have been obtained from a coarse graining procedure that ensure that the water particles at $T=1$ and $\rho=3$ reproduce the compressibility of water at ambient conditions. The surfactant parameters have been obtained from a mapping on Flory-Huggins solubility parameters of ionic surfactants, in which one DPD particle typically corresponds to three carbon atoms in the surfactant chain. We use chain lengths that range from 2 to 7 DPD beads corresponding to experimental chain lengths that range from 6 to 21 carbon atoms. Previous studies of monolayers and bilayers have shown that DP models capture essential features of real systems $[13,17,18]$.

Throughout this Letter, we use reduced units; $r_{c}$ is the unit of length and $k_{B} T$ the unit of energy. In our simulations we used up to 48000 particles at $\rho=3$. The number of surfactants varied from 800 to 1400 and the size of the periodic simulation box was chosen such that the area was approximately $23 \times 23$. The equations of motion are solved using the algorithm of Groot and Warren [15] with a time step of 0.03 .

In the mean-field theories, the number of surfactants per unit area is input and thus fixed. The simulations are more similar to the experimental situation in the following sense: Water and oil particles separate into two phases. Because of periodic boundary conditions there are two interfaces, but the system is large enough for these to be independent of each other. In contrast to the mean-field approach, the surfactants are not constrained to be at the interface but may leave the interface and form (inverse) micelles in the oil or water phase. Although microemulsions are regarded as isotropic one-phase systems, there are oil rich and water rich regions separated by saturated monolayers on a scale corresponding to our simulation box. A two-phase system with very low interfacial tension is therefore a good representation of a real microemulsion. A typical snapshot of the two monolayers is shown in Fig. 1.

Experimentally, it is also difficult to control the number of surfactants at the interface. The experimental data at various chain lengths refer to different surface densities but to similar (very low) interfacial tensions. To mimic the experimental setup, we also perform simulations in which we impose the interfacial tension. At random intervals in the DPD simulation, we perform a Monte Carlo move in which we change the area of the simulation box in such a way that the total volume of the system remains constant. This attempt to change the area is accepted or rejected with a probability given by

$$
\begin{aligned}
\operatorname{acc}(o \rightarrow n)=\min (1, \exp \{ & -\left[U^{n}-U^{o}\right. \\
& \left.\left.\left.-\gamma\left(A^{n}-A^{o}\right)\right] / k_{B} T\right\}\right),
\end{aligned}
$$

where $n$ and $o$ denote the new and old configuration, respectively, $A$ is the area of the simulation box and $U$ is the total potential energy. In both constant area and constant tension ensembles, we could compute an average density of surfactants at the interface, $N / A$, by employing a cluster algorithm to decide which surfactants are at the interface [14]. $A$ is the area of the simulation box, or the average box area in the constant tension simulations. The surface tension was computed via the difference in normal and tangential pressure [19]. The surface tension versus surface density equation of state was the same in the two ensembles.
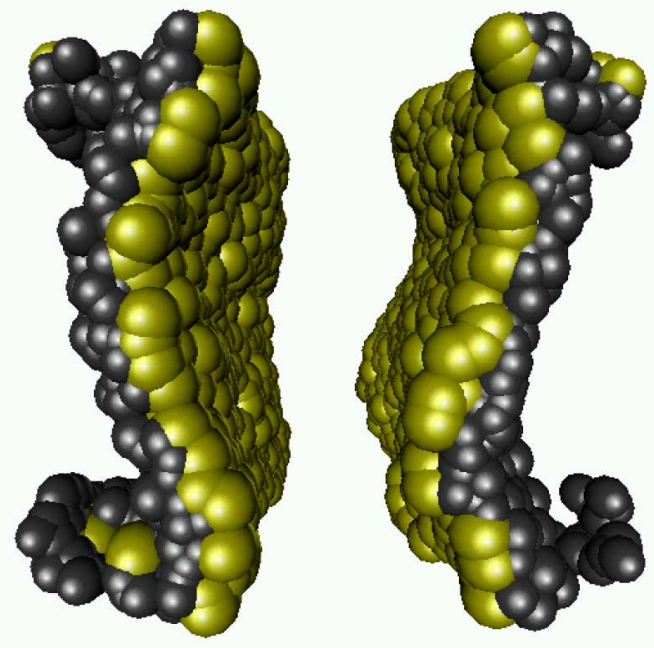

FIG. 1 (color online). Snapshot of two monolayers of ht 4 surfactants at $\gamma=0$. Head beads are in light gray or yellow and tail beads are in dark gray. Water (in the middle) and oil (on the sides) are not shown. 
The height fluctuations of the interface were calculated as in Ref. [14]. Figure 2 shows some typical results of the Fourier analyses. The lines correspond to the fits of Eq. (2) to the results for low wave numbers. The fits give both the interfacial tension and the bending modulus. The data for the interfacial tension correspond nicely to the values that are imposed or computed from the pressure components, indicating that the assumptions behind Eq. (2) are justified. Note that the use of Eq. (2) implies that we assume a zero average curvature of the interface and compute the free energy costs related to deviations from this flat interface. This condition is imposed on the system by the periodic boundary conditions. Experimentally, the asymmetric surfactants that we consider in the simulations may give an interface with a (small) nonzero curvature.

Figure 3 shows the bending modulus for ht ${ }_{n}$ surfactants $(n=2-7)$ as a function of the surface density 3(a) and interfacial tension 3(b). Although we performed relatively long simulations, the scatter in the data is large, illustrating that the bending modulus is difficult to compute accurately [10]. This makes it difficult to compute the chain length dependence of $\kappa$ from simulations at a single surface density or interfacial tension. Figure 3 shows that, by systematically varying the surface density and interfacial tension, we can determine the dependence of $\kappa$ on these properties for various chain lengths. We observe that the bending modulus increases monotonically with the density of surfactants at the interface. Bending the interface becomes increasingly costly because of the packing constraints of the surfactants at the interface. Similar results have been obtained by Laradji and Mouritsen [12].

To determine the chain length dependence at a given surface density or a given interfacial tension, we made linear fits to the results in Fig. 3 for each chain length. From the lines in Fig. 3(a), we computed data points for some densities (shown in Fig. 4, solid symbols). Similarly, the lines in Fig. 3(b) were used to obtain the

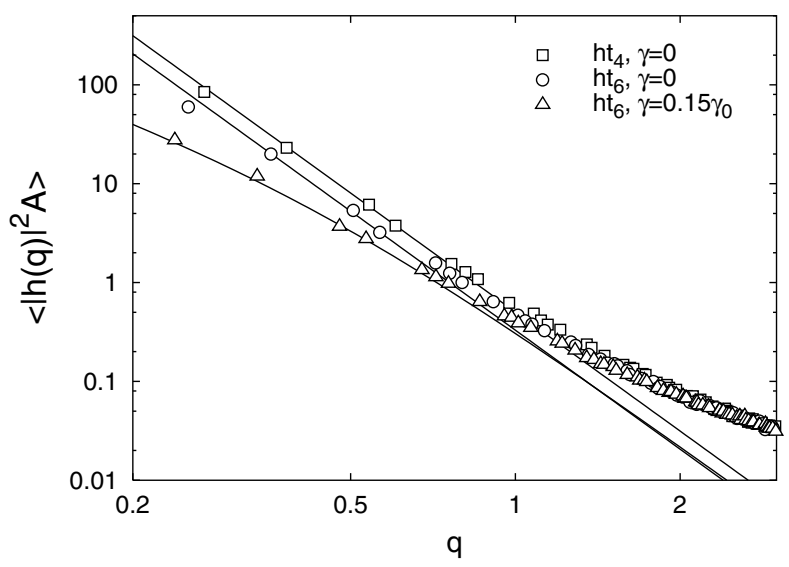

FIG. 2. Fourier spectra for some typical simulations. The lines are fits of the four lowest $q$ values to $1 /\left(\gamma q^{2}+\kappa q^{4}\right)$, where $\gamma$ is the imposed surface tension. $\gamma_{0}$ is the bare oil-water interfacial tension. open symbols in Fig. 4. Each of the data sets in Fig. 4 were fitted to the line $\kappa=a+b n^{p}$. The solid lines are constant density lines and the dashed lines are constant tension lines. Under both conditions, the bending modulus increases with chain length. However, the behavior is qualitatively different when the monolayers have the same surface density and when they have the same interfacial tension. Equal surface densities give $p=1.4-1.6$, while equal interfacial tensions give $p=0.91-0.98$. As the chain length increases, bending the interface becomes increasingly difficult because of packing constraints. However, the surface density will be lower for the longer surfactants at a given interfacial tension. This results in a decrease of the bending modulus, which explains the lower value for $p$ in the case of equal interfacial tensions. In both cases, the exponent is not constant but increases with increasing density and decreasing tension, respectively.

It is instructive to make a more detailed comparison with the experimental data. In our model, a tail bead corresponds to approximately three $\mathrm{CH}_{2}$ groups of a surfactant tail. The surfactants shown in Fig. 4 correspond

a)

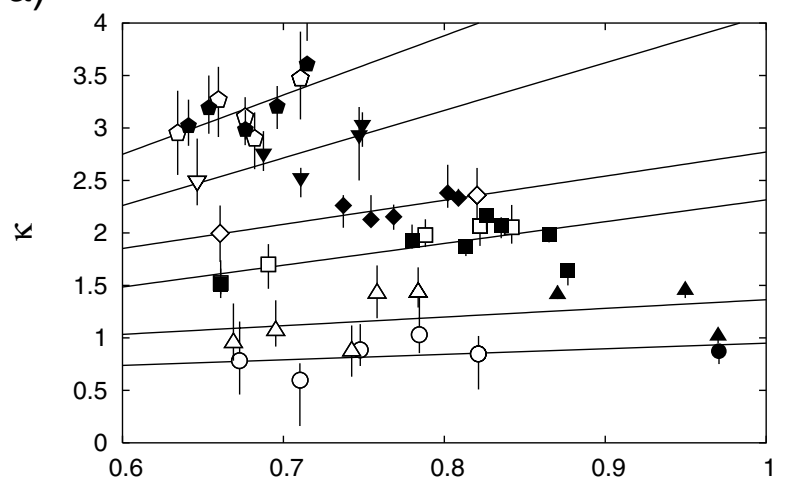

b)

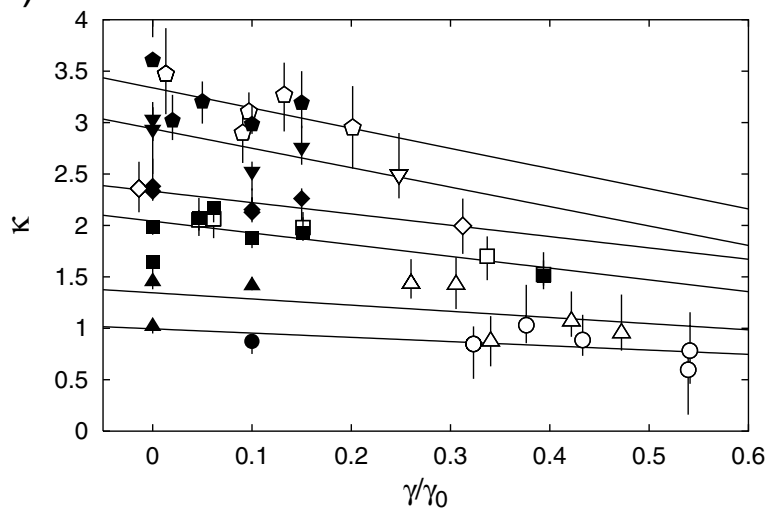

FIG. 3. Bending modulus for $\mathrm{ht}_{n}$ surfactants as a function of surfactant concentration at the interface (a) and interfacial tension (b). $\bigcirc, n=2 ; \triangle, n=3 ; \square, n=4 ; \diamond, n=5 ; \nabla, n=6$, pentagon, $n=7$. Open symbols denote data from constant area simulations and solid symbols denote data from constant interfacial tension simulations. The lines are linear fits to the data for each chain length. 


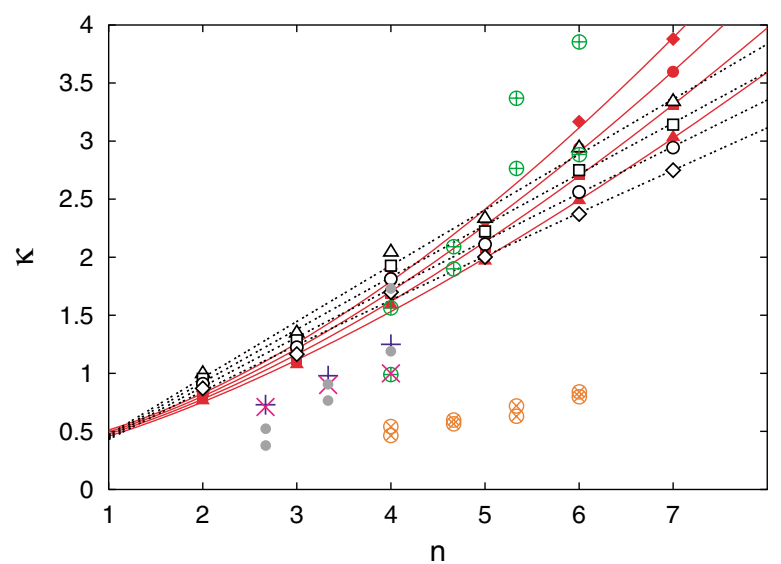

FIG. 4 (color online). Bending modulus as a function of chain length. The solid symbols are for $N / A=0.65(\triangle), N / A=0.70$ $(\square), N / A=0.75(\bigcirc)$, and $N / A=0.80(\diamond)$. The open symbols are for $\gamma=0(\triangle), \gamma=0.1 \gamma_{0}(\square), \gamma=0.2 \gamma_{0}(\bigcirc)$, and $\gamma=$ $0.3 \gamma_{0}(\diamond)$. The lines are fits to $\kappa=a+b n^{p}$. Solid lines are constant density lines and dashed lines are constant tension lines. The points are experimental values: $\oplus$ denotes $\kappa+\bar{\kappa} / 2$ for $C_{i}$ DMAO (amine oxides) from Ref. [6]. $\otimes$ denotes $\kappa+\bar{\kappa} / 2$ for $\mathrm{C}_{i}-\mathrm{C}_{12}$ dialkylammoniumbromides from Ref. [9]. + denotes $\kappa$ for $\mathrm{C}_{i} \mathrm{E}_{4}$ and $\times$ for $\mathrm{C}_{i} \mathrm{E}_{5}$ (polyethyleneglycol alkyl ethers) from Ref. [20]. denotes $\kappa+\bar{\kappa} / 2$ for $C_{i} E_{5}$ from Ref. [6]. We used the mapping $n=i / 3$.

therefore to chains with 6-21 alkyl units. Experimentally, the bending modulus has been determined for chains with 8 -18 alkyl units $[5,6,9,20]$. In Fig. 4, we plotted data from Refs. $[6,9,20]$ that have three or more chain lengths for a given head group and oil type. It is remarkable that our simple model gives such a good estimate of $\kappa$. It reproduces not only the qualitative behavior, but also the order of magnitude (in units of $k_{B} T$ ). Gradzielski et al. found $\kappa+\bar{\kappa} / 2 \propto n^{2.95}$ [6]. This appears to be in contrast with our simulation, which shows that at constant interfacial tension, $p \approx 1$. However, if we distinguish between data with different head groups, $p \approx 1$ seems to be an equally good estimate also for the experimental data. Accurate data for a wider chain length range would be required to confirm our hypothesis that experiments at constant interfacial tension will yield an exponent that is significantly lower than that predicted by mean-field theories at constant density.

Finally, we remark that also our exponent $p$ at fixed density is lower than the theoretical predictions $[1,8]$. With three $\mathrm{CH}_{2}$ groups per bead, $r_{c}=6.5 \AA$ [17] and we get areas per molecule in the range $40-60 \AA^{2}$ at $\gamma \approx 0$, depending on the chain length. The theoretical calculations by Würger show that, only at very low surface areas, we would expect $p=3$ [4]. However, as we study a selfassembled monolayer, such densities are inaccessible in practice.

In this Letter, we have shown that molecular simulations using a mesoscopic oil-water-surfactant system can be used to systematically investigate the effect of changes in the surfactant structure on the bending modulus. We confirm a power-law variation with chain length. However, our simulations indicate that the exponent of this power law depends crucially on the experimental conditions. Since the experiments have been performed at different conditions than for which the theoretical predictions have been made, the apparent agreement of the experimental and theoretically predicted exponent might not hold. Further study is required to determine whether this deviation is related to differences in the models being considered or to the underlying assumptions in the theory.

These investigations are supported in part by the Norwegian Research Council (Grant No. 145184/432), by the Netherlands Research Council for Chemical Sciences $(\mathrm{CW})$, and by the Netherlands Organization for Scientific Research (NWO) through PIONIER.

*Author to whom correspondence should be addressed. Electronic address: live.rekvig@phys.chem.ntnu.no

[1] S. A. Safran, Statistical Thermodynamics of Surfaces, Interfaces, and Membranes (Addison-Wesley, Reading, MA, 1994).

[2] T. Hellweg and D. Langevin, Phys. Rev. E 57, 6825 (1998).

[3] I. Szleifer, D. Kramer, A. Ben-Shaul, D. Roux, and W. M. Gelbart, Phys. Rev. Lett. 60, 1966 (1988).

[4] A. Würger, Phys. Rev. Lett. 85, 337 (2000).

[5] M. Gradzielski, D. Langevin, and B. Farago, Phys. Rev. E 53, 3900 (1996).

[6] M. Gradzielski, D. Langevin, T. Sottmann, and R. Strey, J. Chem. Phys. 106, 8232 (1997).

[7] C. R. Safinya, E. B. Sirota, D. Roux, and G. S. Smith, Phys. Rev. Lett. 62, 1134 (1989).

[8] I. Szleifer, D. Kramer, A. Ben-Shaul, W. M. Gelbart, and S. A. Safran, J. Chem. Phys. 92, 6800 (1990).

[9] J. Eastoe, D. Sharpe, R. K. Heenan, and S. Egelhaaf, J. Phys. Chem. B 101, 944 (1997).

[10] R. Goetz, G. Gompper, and R. Lipowsky, Phys. Rev. Lett. 82, 221 (1999).

[11] E. Lindahl and O. Edholm, Biophys. J. 79, 426 (2000).

[12] M. Laradji and O. G. Mouritsen, J. Chem. Phys. 112, 8621 (2000).

[13] L. Rekvig, M. Kranenburg, J. Vreede, B. Hafskjold, and B. Smit, Langmuir 19, 8195 (2003).

[14] L. Rekvig, B. Hafskjold, and B. Smit, J. Chem. Phys. 120, 4897 (2004).

[15] R. D. Groot and PB. Warren, J. Chem. Phys. 107, 4423 (1997).

[16] R. D. Groot, Langmuir 16, 7493 (2000).

[17] R. D. Groot and K. Rabone, Biophys. J. 81, 725 (2001).

[18] M. Kranenburg, M. Venturoli, and B. Smit, Phys. Rev. E 67, 060901 (2003).

[19] D. Frenkel and B. Smit, Understanding Molecular Simulations: from Algorithms to Applications (Academic, San Diego, 2002), 2nd ed.

[20] T. Sottmann and R. Strey,J. Chem. Phys. 106, 8606 (1997). 